\title{
c-Myc is crucial for the expression of LAT1 in MIA Paca-2 human pancreatic cancer cells
}

\author{
KEITARO HAYASHI ${ }^{1}$, PROMSUK JUTABHA ${ }^{1}$, HITOSHI ENDOU ${ }^{2}$ and NAOHIKO ANZAI ${ }^{1}$ \\ ${ }^{1}$ Department of Pharmacology and Toxicology, Dokkyo Medical University School of Medicine, 880 Kitakobayashi, \\ Mibu, Tochigi 321-0293; ${ }^{2}$ J-Pharma Co., Ltd., 2-16-8 Shinjuku, Shinjuku-ku, Tokyo 160-0022, Japan
}

Received March 29, 2012; Accepted May 21, 2012

DOI: 10.3892/or.2012.1878

\begin{abstract}
Tumor cells take up a massive amount of nutrition compared to normal cells for increased metabolism. Therefore, special transporters for organic materials are required to satisfy the powerful consumption of nutrition in tumor cells. L-type amino acid transporter 1 (LAT1) incorporates large neutral amino acids, most of which are also categorized as essential amino acids, into cells in a $\mathrm{Na}^{+}$-independent manner. Because of its high expression levels in a variety of cancer cells, it is speculated that LAT1 functions as a key transporter for highly effective delivery of essential amino acids into cancer cells. In this regard, LAT1 inhibitor is expected to have clinical benefit for cancer therapy. However, the molecular mechanism of enrichment of LAT1 in cancer cells remains poorly understood. Here, we show that a proto-oncogene, c-Myc, is a critical positive regulator of LAT1 expression in MIA Paca-2 human pancreatic cancer cells. The uptake of leucine, a representative neutral amino acid, was strictly dependent on LAT1 in MIA Paca-2 cells, and siRNA-mediated knockdown of LAT1 inhibited cell proliferation. Diminished c-Myc expression with siRNA resulted in severe reduction of LAT1 protein levels as well as mRNA levels, which, in turn, led to a significant defect of leucine incorporation. The LAT1 promoter has a canonical c-Myc binding sequence and overexpression of c-Myc increased LAT1 promoter activity, whereas mutation of c-Myc binding site diminished this effect. Our results suggest biological significance of LAT1 in tumor growth and molecular machinery that could explain why LAT1 is preferentially expressed in cancer cells.
\end{abstract}

\section{Introduction}

Cancer cells increase their cellular metabolism rate to produce a high level of energy and for biosynthesis of cellular materials

Correspondence to: Dr Naohiko Anzai, Department of Pharmacology and Toxicology, Dokkyo Medical University School of Medicine, 880 Kitakobayashi, Mibu, Tochigi 321-0293, Japan

E-mail: anzai@dokkyomed.ac.jp

Key words: LAT1, neutral amino acid transporter, c-Myc, human pancreatic cancer cells for continuous proliferation (1). The conversion of glucose metabolism from oxidation to glycolysis is one of the representative strategies for generation of ATP in cancer cells (2). However, in order to meet the high metabolic demand, cancer cells must boost the processes for high level of uptake of nutrition sources such as glucose and amino acids. Indeed, it has been shown that the expression of GLUT1, a transporter for glucose, is promoted in cancer cells $(3,4)$. Expression of glutamine transporters such as ASCT2 is also facilitated $(5,6)$, and this would support the cellular abundance of glutamine, which is consumed as a source of nitrogen and energy in tumor cells (7). The coordination of expression of highly efficient transporters for organic materials is requisite for powerful consumption of resources to ensure sustained cancer cell proliferation (8), and disruption of this system would lead to failure of tumor growth.

L-type amino acid transporter 1 (LAT1) is a member of the solute carrier (SLC) family and incorporates neutral amino acids into cells in a $\mathrm{Na}^{+}$-independent manner (9). Whereas the normal body displays low levels and restricted expression of LAT1, high levels of LAT1 expression have been observed in a wide variety of cancer cells $(9,10)$. Therefore, it is speculated that LAT1 has a pivotal role in growth of tumor cells by promoting uptake of essential amino acids. Indeed, the LAT1-specific inhibitor JPH203 (KYT0353) attenuated the incorporation of essential amino acids in cancer cell lines and the growth of human tumor cells planted into a nude mouse (11). For these reasons, JPH203 has attracted attention as a potentially effective drug for cancers with less toxicity, especially for therapy of tumors with extremely poor prognosis such as pancreatic cancer and gastric scirrhous cancer. However, the molecular mechanism underlying the predominant enrichment of LAT1 in cancer cells has remained unclear.

Here we defined c-Myc, a proto-oncogene that encodes a transcription factor, as a critical regulator for LAT1 expression in MIA Paca-2 human pancreatic cancer cells, which require LAT1 for uptake of neutral amino acids. The LAT1 promoter has a canonical c-Myc binding sequence, and knockdown of c-Myc mediated by siRNA resulted in a reduction of LAT1, leading to disturbed neutral amino acid incorporation. Moreover, overexpression of c-Myc increased LAT1 promoter activity, whereas this effect was not observed with mutation of c-Myc binding site. Taken together, these results indicate that c-Myc plays a pivotal role for accelerated expression of LAT1 in human pancreatic cancer cells. 


\section{Materials and methods}

Cells. MIA Paca-2 cells were purchased from Japan Health Sciences Foundation (Tokyo, Japan) and maintained in Dulbecco's modified Eagle's medium supplemented with $10 \%$ fetal bovine serum, $2 \mathrm{mM}$ L-glutamine, $0.1 \mathrm{mM}$ non-essential amino acids, and penicillin/streptomycin.

Antibodies. Anti-LAT1 antibody was described previously (12). Anti-c-Myc antibody (9E11) was purchased from Medical and Biological Laboratories (Nagoya, Japan). Anti-GAPDH (rabbit polyclonal) was purchased from Sigma-Aldrich (St. Louis, MO, USA).

siRNA transfection. Silencer select control siRNA (\#1 and 2), pre-designed c-Myc-specific siRNA (s9129 and s9130) and LAT1-specific siRNA (s15653 and s15655) were purchased from Ambion (Austin, TX, USA). siRNA was transfected into cells seeded in a 24-well plate with Lipofectamine RNAi MAX (Invitrogen, Carlsbad, CA, USA). Cells were cultured for 3 days to knockdown endogenous proteins and used for further experiments.

Western blot analysis. Cells were lysed in SDS sample buffer and boiled.Cell lysate samples were resolved by SDS-polyacrylamide gel electrophoresis and transferred to a PVDF membrane. Immunoblotting was performed with a standard protocol.

Real-time (RT) PCR. Total RNA was extracted using an RNeasy mini kit (Qiagen, Valencia, CA, USA). The cDNA was synthesized from total RNA with a prime-script RT reagent kit (Takara Bio., Shiga, Japan). RT-PCR was performed using SYBR Premix Ex Taq (Takara Bio.). The primers for RT-PCR (HA097381-F and -R for LAT1, HA067812-F and -R for GAPDH) were purchased from Takara Bio. The relative amount of LAT1 mRNA was normalized to GAPDH.

$\left[{ }^{14} \mathrm{C}\right]$-L-leucine uptake. siRNA-transfected cells were cultured for 3 days. The growth medium was removed and cells were washed 3 times with $\mathrm{Na}^{+}$-free Hank's balanced salt solution (HBSS) containing $125 \mathrm{mM}$ choline-Cl, $25 \mathrm{mM}$ HEPES, $4.8 \mathrm{mM} \mathrm{KCl}, 1.2 \mathrm{mM} \mathrm{MgSO}_{4}, 1.2 \mathrm{mM} \mathrm{KH}_{2} \mathrm{PO}_{4}, 1.3 \mathrm{mM} \mathrm{CaCl}_{2}$ and $5.6 \mathrm{mM}$ glucose $(\mathrm{pH}$ 7.4) and further incubated in the same solution at $37^{\circ} \mathrm{C}$ for $\left.10 \mathrm{~min} .{ }^{14} \mathrm{C}\right]-\mathrm{L}$-leucine uptake was initiated by incubating the cells in $\mathrm{Na}^{+}$-free HBSS containing $1.0 \mu \mathrm{M}$ $\left[{ }^{14} \mathrm{C}\right]$-L-leucine (Moravek, Brea, CA, USA) at $37^{\circ} \mathrm{C}$ for $1 \mathrm{~min}$. Uptake was terminated by washing the cells 3 times with icecold $\mathrm{Na}^{+}$-free HBSS. Cells were lysed with $0.1 \mathrm{~N} \mathrm{NaOH}$ and radioactivity was measured using an LSC-5100 $\beta$-scintillation counter (Aloka, Tokyo, Japan). An aliquot of cell lysate was used to determine protein concentration by the BCA protein assay (Pierce Biotechnology, Rockford, IL, USA).

Reporter assay. Human LAT1 promoter fragment (-294/+1 relative to the translation initiation site) was obtained by the PCR method using human genome DNA as a template. The DNA fragment was placed in the polylinker site of pGL3 basic vector (Promega, Madison, WI, USA). A construct with mutation in the c-Myc consensus binding site (located at -210 bp relative to translation initiation site) was generated by replacing the sequence
CACGTG with GGGTC by the PCR method using pGL3 LAT1 (1-294) as a template. c-Myc expression vector pCMV6 c-Myc was purchased from OriGene Technologies (Rockville, MD, USA). For reporter assays, DNA constructs were transfected into MIA PaCa-2 cells with FuGENE HD (Roche Applied Science, Indianapolis, IN, USA) and cells were cultured for $20 \mathrm{~h}$ and then harvested, and luciferase activity was assessed using an Enhanced Luciferase Assay kit (Becton-Dickinson, San Diego, CA, USA). The luciferase activity was normalized to the activity of cotransfected CMV- $\beta$-galactosidase (Invitrogen).

Statistical analysis. All statistical significance was tested with Student's t-test. In siRNA transfection experiments, samples treated with siRNA for gene knockdown were compared to both control-1 and control-2 siRNA-treated samples.

\section{Results}

LAT1 is indispensable for incorporation of neutral amino acid in MIA Paca-2 cells. MIA Paca-2 cells are human pancreatic cancer cells that express a high level of LAT1. To address the functional significance of LAT1 in these cells, we initially evaluated the effect of LAT1 reduction by using siRNA on the uptake of L-leucine, a representative neutral amino acid. We transfected siRNA specific for LAT1 mRNA with two different sequences to exclude targeting any genes other than LAT1 and analyzed $\left[{ }^{14} \mathrm{C}\right]$-labeled $\mathrm{L}$-leucine uptake. The incorporation of $\left[{ }^{14} \mathrm{C}\right]$-L-leucine was severely impaired by knockdown of LAT1 (Fig. 1A). This result indicates that LAT1 is absolutely required for neutral amino acid uptake in MIA Paca-2 cells.

Since cancer cells are continuously proliferating, insufficiency of amino acids resulting from disturbed expression of their transporters would limit the growth of cells. Given that MIA Paca- 2 cells rely on LAT1 for uptake of neutral amino acids, we investigated whether disturbed LAT1 expression results in inhibition of cell proliferation. MIA Paca-2 cells were transfected with LAT1 siRNA and the number of cells was counted. Cell proliferation was markedly impaired by LAT1 siRNA with both sequences (Fig. 1B), indicating that deprivation of neutral amino acids by decreased LAT1 expression leads to inhibition of tumor growth.

Taken together, these results suggest that LAT1 is an essential transporter to provide sufficient neutral amino acids that are crucial for continuous proliferation of human pancreatic cancer cells.

c-Myc is crucial for expression of LAT1. In many cases, the promoter region for gene expression is observed adjacent to the transcriptional starting point. In line with this notion, it has been shown that the LAT1 gene promoter is located at a region of $\sim 300$ bp upstream of the LAT1 translational site (13). Indeed, this region has strong ability as a promoter to drive the expression of posterior cDNA coding luciferase in MIA Paca-2 cancer cells (Fig. 2).

To identify the factors that regulate transcription of the LAT1 gene, we analyzed the sequence of the LAT1 promoter and found that there is a consensus c-Myc binding sequence (CACGTG) $(14,15)$ located 210 bp upstream of the LAT1 translation site. This finding prompted us to examine whether c-Myc participates in the regulation of LAT1 expression in cancer cells, since c-Myc 
A

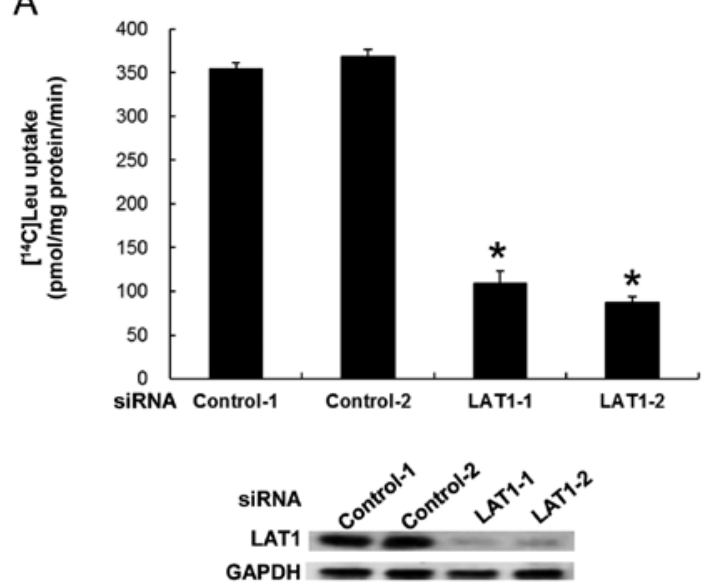

B

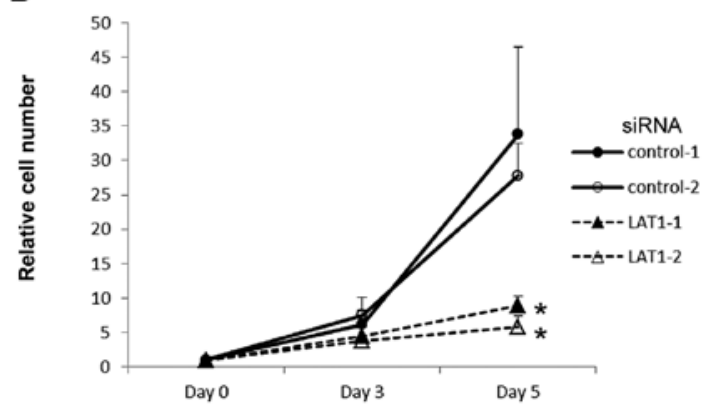

Figure 1. MIA Paca-2 cells depend on LAT1 for incorporation of essential neutral amino acid. (A) Impaired incorporation of essential neutral amino acid by knockdown of LAT1. siRNA for knockdown of LAT1 or control siRNA was transfected into MIA Paca-2 cells and the cells were cultured for 3 days. $\left[{ }^{14} \mathrm{C}\right]$-L-leucine was added and uptake was determined by measuring radioactivity. The knockdown of LAT1 protein was confirmed by western blotting. Data are expressed as the mean \pm SD. ${ }^{*} \mathrm{P}<0.001$. (B) Knockdown of LAT1 leads to growth arrest of tumor cells. MIA Paca-2 cells were transfected with LAT1 siRNA and cultured for indicated days. The number of the cells was counted, and relative cell number to day 1 is shown in the graph. Data expressed as mean $\pm \mathrm{SD} .{ }^{*} \mathrm{P}<0.05$.

is a proto-oncogene that has strong ability to enforce malignant alteration. To address this, we transfected siRNA for knockdown of c-Myc expression into MIA Paca-2 cells and analyzed LAT1 protein level. As shown in Fig. 3A, suppression of c-Myc expression by siRNA with two different sequences dramatically decreased the expression of LAT1 protein. This result indicates
A

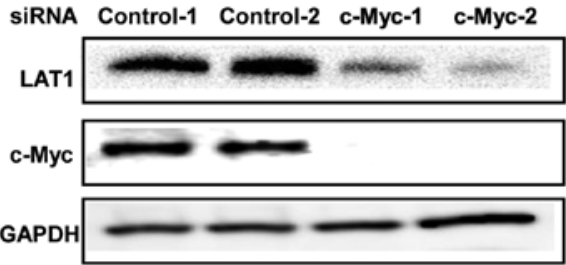

B

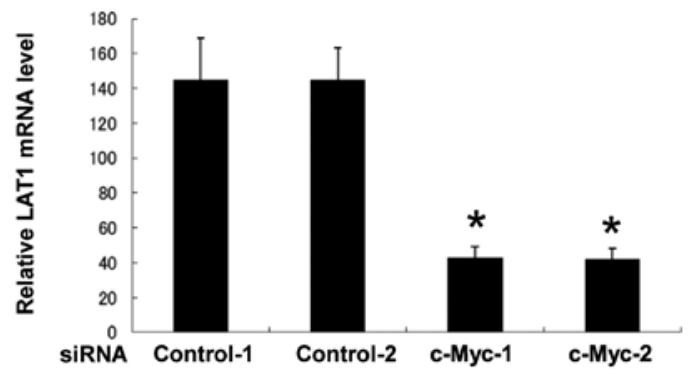

Figure 3. c-Myc is critical for expression of LAT1 in MIA Paca-2 cells. (A) MIA Paca-2 cells were transfected with siRNA for knockdown of c-Myc or a control and cultured for 3 days. Cells were lysed and western blotting was performed with antibodies for LAT1, c-Myc and GAPDH. (B) MIA Paca-2 cells were transfected with c-Myc siRNA and cultured for 3 days. Total RNA was extracted from cells and cDNA was synthesized. Real-time PCR was performed with primers for LAT1 and GAPDH. The amount of LAT mRNA was normalized by GAPDH mRNA. Data are expressed as the mean $\pm \mathrm{SD}$. ${ }^{*} \mathrm{P}<0.005$.

that c-Myc is critical for up-regulation of LAT1 expression in MIA Paca-2 cancer cells.

To determine whether reduction of LAT1 protein expression induced by c-Myc siRNA is derived from a reduced amount of LAT1 mRNA, we performed RT-PCR using RNA from MIA Paca-2 cells transfected with c-Myc siRNA. We found that transfection of c-Myc siRNA resulted in reduction of LAT1 mRNA expression (Fig. 3B). This result suggests that c-Myc regulates LAT1 expression at the mRNA level.

Down-regulation of LAT1 by c-Myc knockdown impairs the uptake of neutral amino acid. Based on our finding that LAT1 is essential for incorporation of neutral amino acids in MIA Paca-2 cells, we postulated that reduction of LAT1 expression caused by knockdown of c-Myc protein could affect the efficiency of neutral amino acid incorporation. To test this hypothesis, we

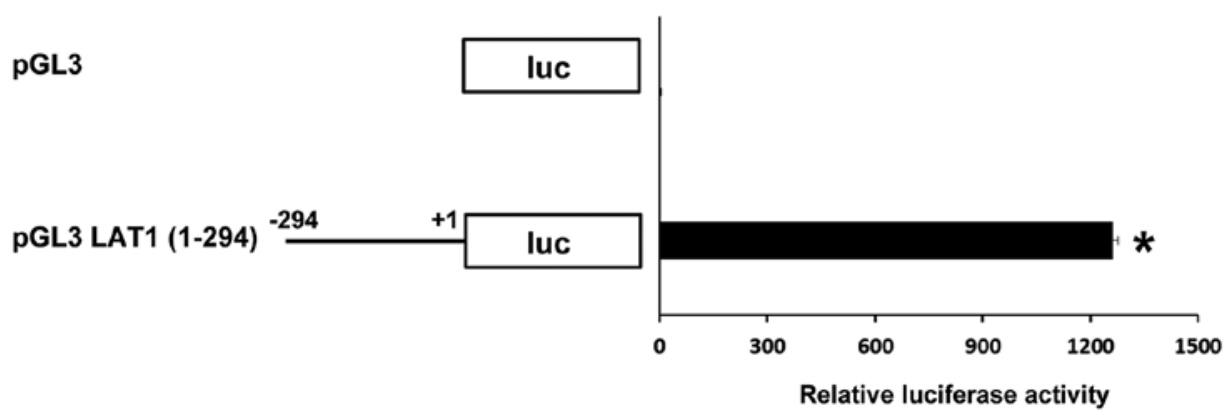

Figure 2. Proximal region of the translational site in the LAT1 gene has promoter activity. pGL3 LAT1 (1-294) or its control vector (pGL3) was transfected into MIA Paca- 2 cells and luciferase activity was analyzed. Data expressed as mean \pm SD. ${ }^{*} \mathrm{P}<0.001$. 


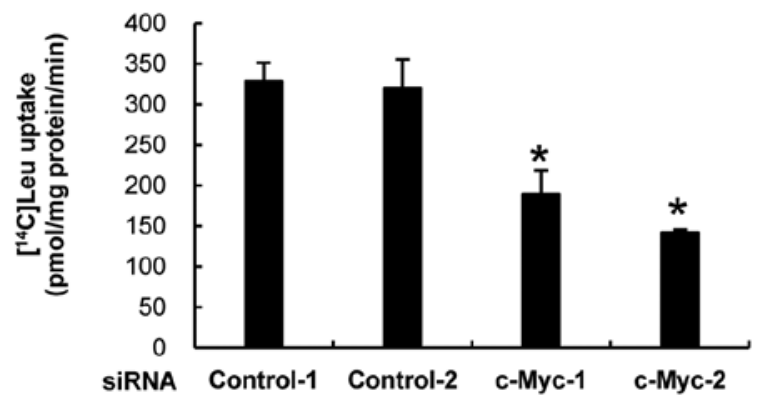

Figure 4. Amino acid uptake is impaired by knockdown of c-Myc. MIA Paca-2 cells were transfected with c-Myc siRNA and cultured for 3 days. $\left[{ }^{14} \mathrm{C}\right]-\mathrm{L}-$ leucine uptake was measured. Data expressed as mean $\pm \mathrm{SD}$. ${ }^{*} \mathrm{P}<0.001$.

transfected c-Myc siRNA into MIA Paca-2 cells and measured $\left[{ }^{14} \mathrm{C}\right]$-labeled leucine uptake. c-Myc siRNA-transfected cells had less ability than that of control siRNA-transfetcted cells for uptake of leucine (Fig. 4). This result indicates that c-Mycmediated up-regulation of LAT1 expression is strictly required to maintain the maximum level of amino acid incorporation in MIA Paca-2 cancer cells.

Consensus c-Myc binding sequence is required for maximum activity of LAT1 promoter. As described above, a consensus c-Myc binding sequence is present in the LAT1 promoter. In addition, our studies demonstrated that reduction of c-Myc results in declined LAT1 expression at the mRNA level. These observations suggest that c-Myc can drive transcription of the LAT1 gene by enhancing the LAT1 promoter activity. To examine this, we performed a reporter assay driven by the LAT1 promoter. The construct for reporter assay used in Fig. 2 and a vector for overexpression of c-Myc were co-transfected into MIA Paca-2 cells and luciferase activity was analyzed. The overexpression of c-Myc increased LAT1 promoter activity (Fig. 5). On the other hand, mutation in the c-Myc binding sequence in LAT1 promoter resulted in significant reduction of promoter activity. In addition, overexpression of c-Myc could not promote activity of LAT1 promoter with mutation in c-Myc binding site. These results indicate that c-Myc enhances LAT1 promoter activity and a consensus binding sequence of c-Myc is crucial for maximum activity of LAT1 promoter.

\section{Discussion}

We have demonstrated the physiological importance of LAT1 as an essential factor to transport neutral amino acids into MIA Paca-2 human pancreatic cancer cells. Inhibition of LAT1 expression by siRNA severely decreased neutral amino acid incorporation, leading to suppression of cell growth. We also demonstrated that knockdown of c-Myc, a proto-oncogene, resulted in a reduction of LAT1 protein as well as mRNA, which led to impaired neutral amino acid incorporation. Moreover, mutation of the c-Myc binding site in the LAT1 promoter region suppressed maximum promoter activity and diminished the effect of c-Myc overexpression. These results indicate that c-Myc is a crucial transcription factor for up-regulation of LAT1 in human pancreatic cancer cells.

In order to satisfy rapid cellular metabolism, cancer cells must take up as much nutrients as possible. Therefore, it is a huge advantage to make utmost use of special molecular devices such as highly effective transporters to maximize the efficiency of nutrition incorporation. Insufficient nutrition resulting from inability to use facilitative transporters would limit the proliferation of cancer cells. Consistent with this notion, we revealed that siRNA-mediated LAT1 reduction remarkably impaired uptake of neutral amino acids, which subsequently induced arrest of the growth of pancreatic cancer cells. Although previous studies using a potential LAT1-specific inhibitor have shown the ability of this drug to inhibit tumor growth (11). Evidence that excludes any effect of this drug on molecules other than LAT1 has not been obtained. Our results obtained by using LAT1 siRNA are important in that they strongly support the drug model of LAT1 being essential for delivery of neutral amino acids into cancer cells.

Results of many studies have shown that LAT1 exhibits preferred expression in cancer cells $(10,12,16-18)$. However, the molecular machinery regulating LAT1 expression in cancer cells has remained a long-standing puzzle. In this study, we showed that c-Myc is a critical factor for promotion of LAT1 expression in human pancreatic cancer cells. Our observation about regulation of LAT1 by virtue of a proto-oncogene would provide a clue that accounts for the high expression level of LAT1 in cancer cells. Many genes associated with the cell cycle such as cyclins are representative targets of c-Myc (19-21). However, c-Myc also enhances biosynthesis as well as energy

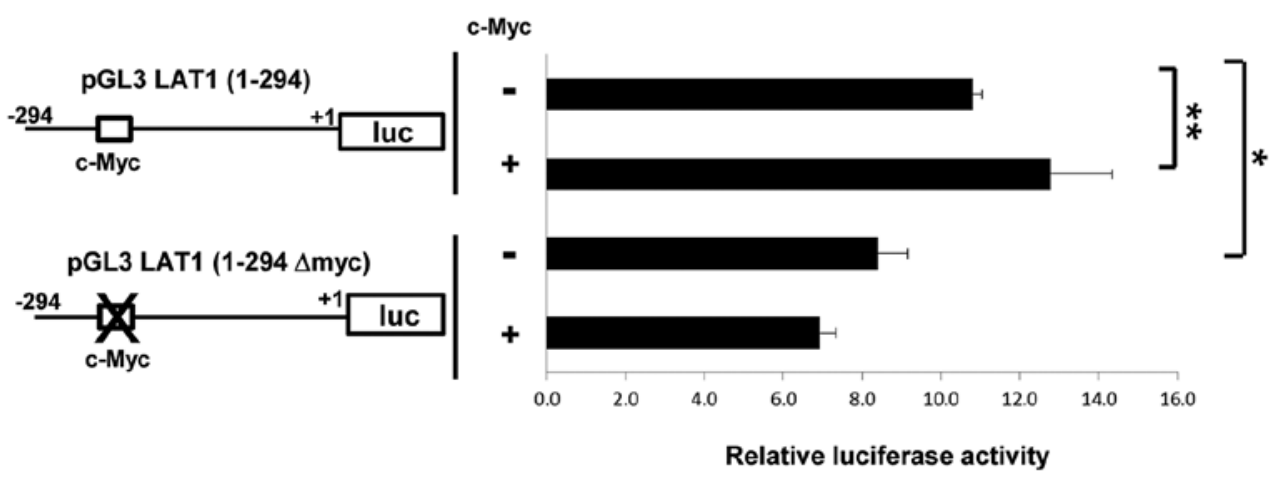

Figure 5. Promoter activity of LAT1 is decreased by mutation in the c-Myc consensus binding sequence. pGL3 LAT1 (1-294) or its c-Myc binding site mutant [pGL3 LAT1 (1-294) $\Delta \mathrm{Myc}$ ] was transfected into MIA Paca-2 cells with or without vector for c-Myc overexpression and luciferase activity was analyzed. ${ }^{*}<0.01$, ${ }_{* * *} \mathrm{P}<0.07$. 
generation. For example, expression of genes required for glucose transport and the glycolytic pathway is up-regulated by c-Myc $(22,23)$. Therefore, c-Myc conducts tumorigenesis by controlling the expression of genes involved in the cell cycle in concert with metabolic genes, and such ability to concurrently regulate a set of genes all required for cell proliferation is an enormous contribution to stable growth of cancer cells. Our results showed that LAT1 is also a c-Myc target as a central transporter of essential neutral amino acids expressed in human pancreatic cancer cells.

Since overexpression of c-Myc enhanced the LAT1 promoter activity and mutation of c-Myc binding site diminished this effect, one possible explanation for up-regulation of LAT1 by c-Myc is its binding to consensus sequence in LAT1 promoter and driving the transcription of LAT1 mRNA. However, although mutation of the c-Myc binding sequence significantly reduced LAT1 promoter activity, the effect was slight. Therefore, we cannot exclude the possibility that c-Myc regulates the mRNA level of LAT1 by some other mechanisms in addition to direct binding to the promoter region. For example, since one of the targets regulated by c-Myc is microRNA (24), c-Myc might increase LAT1 expression by down-regulating microRNA, which interacts with and diminishes LAT1 mRNA. Alternatively, because c-Myc controls some of target gene expression epigenetically $(25,26)$, c-Myc might regulate LAT1 gene expression by modulating chromatin conformations of LAT1 promoter. Further study is required to decide more details of the mechanism for regulation of LAT1 by c-Myc.

In conclusion, we identified c-Myc as a crucial regulator for expression of LAT1. Our results will be helpful in investigation and further development of drugs that target LAT1 more effectively in cancer therapy.

\section{Acknowledgements}

This work was supported in part by grants of TR Project from the New Energy and Industrial Technology Development Organization (NEDO), Japan.

\section{References}

1. DeBerardinis RJ, Lum JJ, Hatzivassiliou G and Thompson CB The biology of cancer: metabolic reprogramming fuels cell growth and proliferation. Cell Metab 7: 11-20, 2008.

2. Warburg O: On the origin of cancer cells. Science 123: 309-314, 1956.

3. Airley RE and Mobasheri A: Hypoxic regulation of glucose transport, anaerobic metabolism and angiogenesis in cancer: novel pathways and targets for anticancer therapeutics. Chemotherapy 53: 233-256, 2007.

4. Macheda ML, Rogers S and Best JD: Molecular and cellular regulation of glucose transporter (GLUT) proteins in cancer. J Cell Physiol 202: 654-662, 2005.

5. Witte D, Ali N, Carlson N and Younes M: Overexpression of the neutral amino acid transporter ASCT2 in human colorectal adenocarcinoma. Anticancer Res 22: 2555-2557, 2002.

6. Li R, Younes M, Frolov A Wheeler TM, Scardino P, Ohori M and Ayala G: Expression of neutral amino acid transporter ASCT2 in human prostate. Anticancer Res 23: 3413-3418, 2003.
7. Levine AJ and Puzio-Kuter AM: The control of the metabolic switch in cancers by oncogenes and tumor suppressor genes. Science 330: 1340-1344, 2010.

8. Ganapathy V, Thangaraju M and Prasad PD: Nutrient transporters in cancer: relevance to Warburg hypothesis and beyond. Pharmacol Ther 121: 29-40, 2009.

9. Kanai Y, Segawa H, Miyamoto K, Uchino H, Takeda E and Endou H: Expression cloning and characterization of a transporter for large neutral amino acids activated by the heavy chain of 4F2 antigen (CD98). J Biol Chem 273: 23629-23632, 1998.

10. Yanagida O, Kanai Y, Chairoungdua A, et al: Human L-type amino acid transporter 1 (LAT1): characterization of function and expression in tumor cell lines. Biochim Biophys Acta 1514: 291-302, 2001.

11. Oda K, Hosoda N, Endo H, et al: L-type amino acid transporter 1 inhibitors inhibit tumor cell growth. Cancer Sci 101: 173-179, 2010.

12. Ichinoe M, Mikami T, Yoshida T, et al: High expression of L-type amino-acid transporter 1 (LAT1) in gastric carcinomas: comparison with non-cancerous lesions. Pathol Int 61: 281-289, 2011.

13. Nii T, Segawa H, Taketani Y, et al: Molecular events involved in up-regulating human $\mathrm{Na}^{+}$-independent neutral amino acid transporter LAT1 during T-cell activation. Biochem J 358: 693-604, 2001.

14. Blackwell TK, Kretzner L, Blackwood EM, Eisenman RN and Weintraub H: Sequence-specific DNA binding by the c-Myc protein. Science 250: 1149-1151, 1990.

15. Alex R, Sözeri O, Meyer S and Dildrop R: Determination of the DNA sequence recognized by the bHLH-zip domain of the N-Myc protein. Nucleic Acids Res 20: 2257-2263, 1992.

16. Sakata T, Ferdous G, Tsuruta T, et al: L-type amino-acid transporter 1 as a novel biomarker for high-grade malignancy in prostate cancer. Pathol Int 59: 7-18, 2009.

17. Kaira K, Oriuchi N, Imai H, et al: Prognostic significance of L-type amino acid transporter 1 (LAT1) and 4F2 heavy chain (CD98) expression in early stage squamous cell carcinoma of the lung. Cancer Sci 100: 248-254, 2009.

18. Kaji M, Kabir-Salmani M, Anzai N, et al: Properties of L-type amino acid transporter 1 in epidermal ovarian cancer. Int J Gynecol Cancer 20: 329-336, 2010.

19. Bouchard C, Thieke K, Maier A, et al: Direct induction of cyclin D2 by Myc contributes to cell cycle progression and sequestration of p27. EMBO J 18: 5321-5333, 1999.

20. Bouchard C, Dittrich O, Kiermaier A, Dohmann K, Menkel A, Eilers $\mathrm{M}$ and Lüscher B: Regulation of cyclin D2 gene expression by the Myc/Max/Mad network: Myc-dependent TRRAP recruitment and histone acetylation at the cyclin D2 promoter. Genes Dev 15: 2042-2047, 2001.

21. Menssen A and Hermeking H: Characterization of the c-Mycregulated transcriptome by SAGE: identification and analysis of c-Myc target genes. Proc Natl Acad Sci USA 99: 6274-6279, 2002.

22. Kim JW,Zeller KI, Wang Y, Jegga AG, Aronow BJ, O'Donnell KA and Dang CV: Evaluation of myc E-box phylogenetic footprints in glycolytic genes by chromatin immunoprecipitation assays. Mol Cell Biol 24: 5923-5936, 2004.

23. Osthus RC, Shim H, Kim S, et al: Deregulation of glucose transporter 1 and glycolytic gene expression by c-Myc. J Biol Chem 275: 21797-21800, 2000 .

24. O'Donnell KA, Wentzel EA, Zeller KI, Dang CV and Mendell JT: c-Myc-regulated microRNAs modulate E2F1 expression. Nature 435: 839-843, 2005.

25. Frank SR, Schroeder M, Fernandez P, Taubert S and Amati B: Binding of c-Myc to chromatin mediates mitogen-induced acetylation of histone H4 and gene activation. Genes Dev 15: 2069-2082, 2001.

26. Frank SR, Parisi T, Taubert S, et al: Myc recruits the TIP60 histone acetyltransferase complex to chromatin. EMBO Rep 4: 575-580, 2003. 